Check for updates

Cite this: RSC Adv., 2017, 7, 36288

Received 5th June 2017

Accepted 15th July 2017

DOI: $10.1039 / \mathrm{c} 7 \mathrm{ra06261c}$

rsc.li/rsc-advances

\section{Unique band structure enhanced visible light photocatalytic activity of phosphorus-doped BiOl hierarchical microspheres $\dagger$}

\begin{abstract}
Fen-Qiang Ma, Jing-Wen Yao, Yan-Feng Zhang (DD* and Yu Wei
In this article, phosphorus (P)-doped BiOl hierarchical microspheres were prepared via a facile hydrolytic method at room temperature. The morphology and structure of the samples were characterized by using X-ray powder diffraction, scanning electron microscopy, high-resolution transmission electron microscopy, X-ray photoelectron spectroscopy, Fourier transform infrared spectroscopy, nitrogen sorption diffraction, photoluminescence spectroscopy and UV-visible diffuse spectroscopy. Based on these results, it was concluded that the $P$ doping was beneficial to forming uniform hierarchical microspheres, which consisted of numerous two-dimensional nanosheets with a thickness in the range of $30-40 \mathrm{~nm}$. It is believed that the phosphorus species had been doped into the lattice of $\mathrm{BiOl}$ and formed $\mathrm{Bi}-\mathrm{O}-\mathrm{P}$ bonds. Based on this fascinating characterization, the photocatalytic activity of $\mathrm{P}$-doped $\mathrm{BiOl}$ was evidently improved, and the photocatalytic activity of the $4 \% \mathrm{P}$ - $\mathrm{BiOl}$ sample for degradation of $\mathrm{RhB}$ is almost 2.6 times as high as pure $\mathrm{BiOl}$ in the case of visible light irradiation. Furthermore, the $\mathrm{P}$ doping could not only increase the width of the valence band but also raise the conduction band minimum, which led to the more efficient separation capacity of photogenerated carriers, the higher mobility of the resulting holes and the greater concentration of superoxide ions. In addition, the possible photocatalytic mechanism of $\mathrm{P}$-doped $\mathrm{BiOI}$ microspheres for degrading Rhodamine $\mathrm{B}$ dye was proposed.
\end{abstract}

\section{Introduction}

Environmental problems and water pollution currently attract world-wide attention, ${ }^{1-3}$ and the urgent task is to seek highly active photocatalysts due to their promising applications for degradation of pollutants. Titanium dioxide $\left(\mathrm{TiO}_{2}\right)$ has been extensively studied and utilized to degrade organic pollutants due to its chemical stability, nontoxicity and low cost. ${ }^{4-6}$ However, $\mathrm{TiO}_{2}$ has a wide band gap energy (3.2 and $3.0 \mathrm{eV}$ for anatase and rutile phases, respectively), ${ }^{7}$ which results in the fact that $\mathrm{TiO}_{2}$ is only responsive to the UV region of the solar spectrum. In order to make full use of the richness of solar light, the development of efficient optical photocatalysts is imperative.

To date, many efficient photocatalysts have been explored and shown exciting photocatalytic activity. Bismuth oxyhalide (BiOX) has received persistent attention because of its ability to remove contaminants from aquatic environments. The BiOX $(\mathrm{X}=\mathrm{Cl}, \mathrm{Br}, \mathrm{I})$ is an important $\mathrm{V}-\mathrm{VI}-\mathrm{VII}$ ternary compound in a layered structure comprising of $\left[\mathrm{Bi}_{2} \mathrm{O}_{2}\right]^{2+}$ slabs interleaved

National Demonstration Center for Experimental Chemistry Education, College of Chemistry and Material Science, Hebei Normal University, Shijiazhuang, 050024, P. R. China. E-mail: zhangyanfeng@mail.hebtu.edu.cn; Fax: +86-0311-80788803; Tel: +86-0311-80787400

$\dagger$ Electronic supplementary information (ESI) available. See DOI: 10.1039/c7ra06261c with double slabs of halogen atoms (Fig. 1). Especially, the BiOI has the maximum absorption ability in visible-light region due to its smallest band gap (1.7-1.9 eV). ${ }^{8-10}$ Moreover, the capability of BiOI hierarchical architectures in the oxidative degradation of various organic dyes has been investigated in detail.11-13 Numerous methods have been studied to synthesize BiOI hierarchical microspheres, for example, Wang et al. synthesized

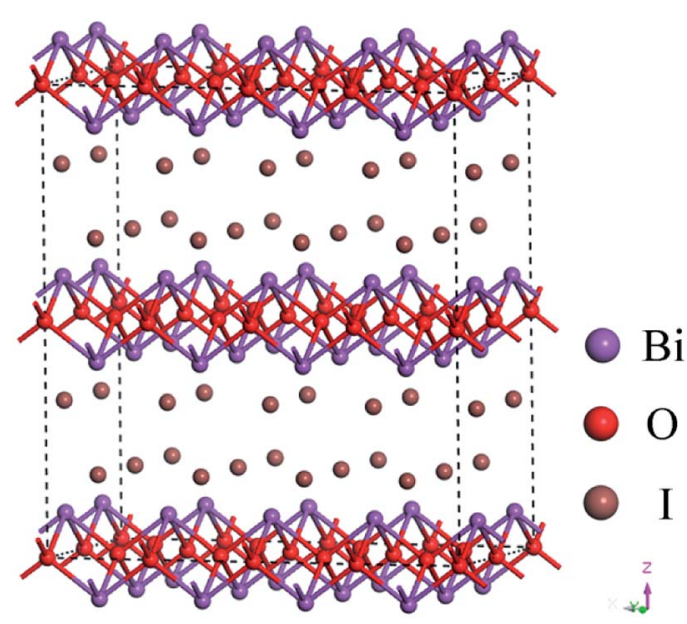

Fig. 1 Schematic illustrations of the crystal structure of BiOl. 
BiOI microspheres via a EG-assisted solvothermal route at $160{ }^{\circ} \mathrm{C}$ for $12 \mathrm{~h}^{14}$ Qin et al. prepared BiOI microspheres using a generalized ionic liquid-assisted solvothermal method. ${ }^{15}$ However, long synthesis time, ${ }^{16}$ high reaction temperature ${ }^{17,18}$ and expensive ionic liquids ${ }^{\mathbf{1 9}}$ are common in synthetic approaches. In addition, it was found that iodine, titanium or ferric doping for BiOI is also effective method to synthesize BiOI hierarchical microspheres, ${ }^{\mathbf{2 0 - 2 2}}$ respectively. However, the photocatalytic activity of the above work needs to be further improved. Thereby, it is imperative to prepare a more efficient photocatalysts by using simple equipment, mostly used reagent and a lower synthesis temperature.

Herein, the P-doped BiOI hierarchical microspheres were prepared by a simple and facile method without making use of expensive reagent and equipment at room temperature. As far as we know, this is the first to report the $\mathrm{P}$ doping for BiOI microspheres. The effects of $\mathrm{P}$ doping on morphology and chemical bonds of BiOI were investigated. In additions, the photocatalytic performance of the P-doped BiOI powders was measured under the visible light irradiation. Moreover, for all we know, there was no one work established a link between the photocatalytic activity and the band structure of BiOI. Consequently, the effects of $\mathrm{P}$ doping on the photocatalytic mechanism of BiOI was proposed.

\section{Experimental}

\subsection{Preparation of BiOI samples}

The chemicals used in this work were of analytical reagent grade without further purification. In a typical synthesis, $3.40 \mathrm{~g}$ of $\mathrm{Bi}\left(\mathrm{NO}_{3}\right)_{3} \cdot 5 \mathrm{H}_{2} \mathrm{O}$ was dissolved in $7.00 \mathrm{~mL}$ of glacial acetic acid with magnetic stirring for $30 \mathrm{~min}$ at room temperature (solution A). At the same time, $1.66 \mathrm{~g}$ of $\mathrm{KI}, 1.64 \mathrm{~g}$ of $\mathrm{CH}_{3} \mathrm{COONa}$ and a certain amount of $\mathrm{NaH}_{2} \mathrm{PO}_{4}$ were dissolved in $10.00 \mathrm{~mL}$ deionized water with ultrasonic irradiation for about $30 \mathrm{~min}$ (solution B). And then, $7.00 \mathrm{~mL}$ of solution A was dropwise added into solution $B$ with magnetic stirring and finally the red solution was gradually formed. After continuously stirring for $3 \mathrm{~h}$ at room temperature, the BiOI sample was collected and washed with deionized water and ethanol absolute several times to remove any ionic residuals, and then dried at $60{ }^{\circ} \mathrm{C}$ for $12 \mathrm{~h}$. The synthesis of pure BiOI was also performed with the same processes, but without the addition of $\mathrm{NaH}_{2} \mathrm{PO}_{4}$ into the reaction solution. The as-prepared samples were named as BiOI, 2\% $\mathrm{P}-\mathrm{BiOI}$, 4\% $\mathrm{P}$-BiOI, 6\% $\mathrm{P}$-BiOI and $8 \% \mathrm{P}$-BiOI corresponding to the molar ratio of $\mathrm{P}$ to $\mathrm{Bi}$ in the synthesis processes.

\subsection{Characterization}

The as-prepared samples were characterized by X-ray powder diffraction (XRD) using a Bruker D8 Advance diffractometer with $\mathrm{Cu} \mathrm{K}_{\alpha}$ radiation $(\lambda=1.5406 \AA)$ in the $2 \theta$ range $10-80^{\circ}$, operated at $40 \mathrm{kV}$ and $40 \mathrm{~mA}$. The morphology of the BiOI samples was observed by $\mathrm{S}-4800$ scanning electron microscope (SEM, Hitachi, Japan) using an accelerating voltage of $10 \mathrm{kV}$. High-resolution transmission electron microscopy (HRTEM) image was taken using field emission transmission electron microscopy (Tecnai G2 F30). X-ray photoelectron spectroscopy (XPS) measurements were performed on a VG Scientific ESCALAB Mark II spectroscopy to determine the binding state of the $\mathrm{Bi}, \mathrm{O}, \mathrm{I}$ and $\mathrm{P}$ ions. The $\mathrm{C}(1 \mathrm{~s})$ level was used as an internal reference at $284.6 \mathrm{eV}$. Fourier-transform infrared (FTIR) spectra of the samples were recorded on FTIR-9800 spectrometer (made in Japan). The surface area was obtained by the BrunauerEmmett-Teller (BET) method, and pore size distribution was calculated from the adsorption branch of the isotherms by the Barrett-Joyner-Halenda (BJH) model. The photoluminescence (PL) spectra of the prepared samples were detected by Hitachi F4600 (made in Japan). The UV-vis DRS spectra were obtained via the UV-vis spectrometer (Hitachi U-3010 spectrophotometer) by diffuse reflectance method with $\mathrm{BaSO}_{4}$ powder as the substrate. The electrochemical measurements were carried out by using a three-electrode system at room temperature (CHI 660E). The electrolyte was $0.1 \mathrm{M} \mathrm{Na}_{2} \mathrm{SO}_{4}$ aqueous solution.

\subsection{Photocatalytic activity measurements}

The photocatalytic activity of the as-prepared samples was evaluated by degradation of the Rhodamine $\mathrm{B}(\mathrm{RhB})$ aqueous solution $\left(20 \mathrm{mg} \mathrm{L}^{-1}\right)$. A $300 \mathrm{~W}$ xenon lamp with a $420 \mathrm{~nm}$ cutoff filter was used as the light source. A total of $0.08 \mathrm{~g}$ of photocatalyst was added into $100 \mathrm{~mL} \mathrm{RhB}$ solution. The temperature of the photocatalytic reaction was kept at $25{ }^{\circ} \mathrm{C}$. Prior to irradiation, the suspension was stirred in the dark for $30 \mathrm{~min}$ to ensure the establishment of an adsorption-desorption equilibrium. During irradiation, $4 \mathrm{~mL}$ suspension was sampled at given time intervals and centrifuged to remove the remaining particles. The residual RhB solution was then analyzed by measuring the maximum absorbance of RhB at $553 \mathrm{~nm}$ using a $752 \mathrm{~N}$ ultraviolet-visible spectrometer.

\section{Results and discussion}

\subsection{XRD analysis}

Fig. 2a shows the XRD patterns of BiOI and P-doped BiOI samples. It is seen that all of the samples can be identified as the tetragonal BiOI (JCPDS no. 10-0445) ${ }^{14,23}$ and well crystallized. In addition, by comparing the (102) and (110) diffraction peaks in the range of $2 \theta=28-33^{\circ}$ as shown in Fig. $2 \mathrm{~b}$, it can be seen that the peaks of P-BiOI slightly shift in the case of the presence of phosphorus. We also find that the other peak positions of P-BiOI move slightly toward to the lower $2 \theta$ values. Furthermore, Table 1 shows the lattice constants of BiOI and Pdoped BiOI samples, which are calculated by (102) XRD peak position of the tetragonal phase. ${ }^{24}$ The analyses show that the crystal lattice parameters of P-BiOI increase with enhancing the doping amount of $\mathrm{P}$ gradually. The enlargement of lattices of $\mathrm{P}$ BiOI may be resulted from the $\mathrm{P}$ species doped into the lattice of BiOI.

\subsection{SEM and HRTEM analyses}

Fig. 3 shows the SEM images and HRTEM image of 4\% P-BiOI. In Fig. S1, $\uparrow$ it can be seen that the BiOI consists of irregularsphere-like structure with the diameter from several to several 

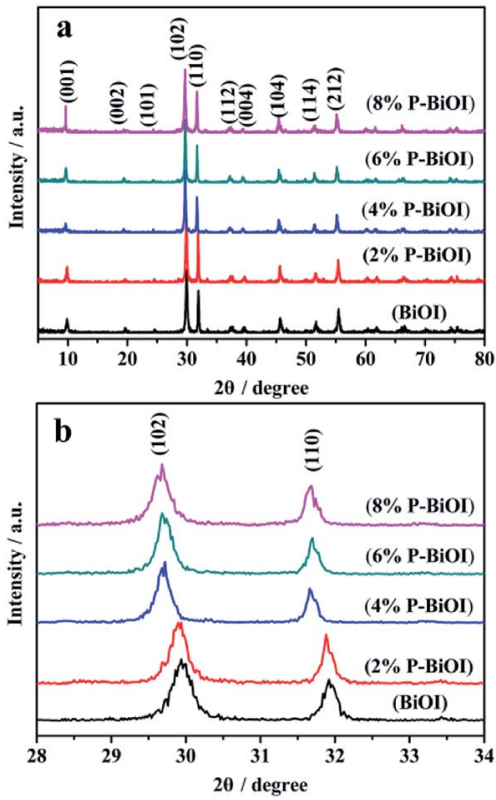

Fig. 2 (a) XRD patterns of $\mathrm{BiOl}$ and P-doped $\mathrm{BiOl}$ samples; (b) diffraction peak positions of the (012) and (110) plane in the range of $2 \theta$ $=28-33^{\circ}$.

Table 1 Crystallographic parameters of $\mathrm{P}$-doped $\mathrm{BiO}$ determined by (102) XRD peak position of the tetragonal phase

\begin{tabular}{llll}
\hline Sample & $a=b(\AA)$ & $c(\AA)$ & Volume of cell $\left(\AA^{3}\right)$ \\
\hline BiOI & 3.9614 & 9.0483 & 141.9972 \\
$2 \%$ P-BiOI & 3.9663 & 9.0700 & 142.6858 \\
$4 \%$ P-BiOI & 3.9902 & 9.1328 & 145.4103 \\
$6 \%$ P-BiOI & 3.9903 & 9.1414 & 145.5573 \\
$8 \%$ P-BiOI & 3.9910 & 9.1646 & 146.0246
\end{tabular}
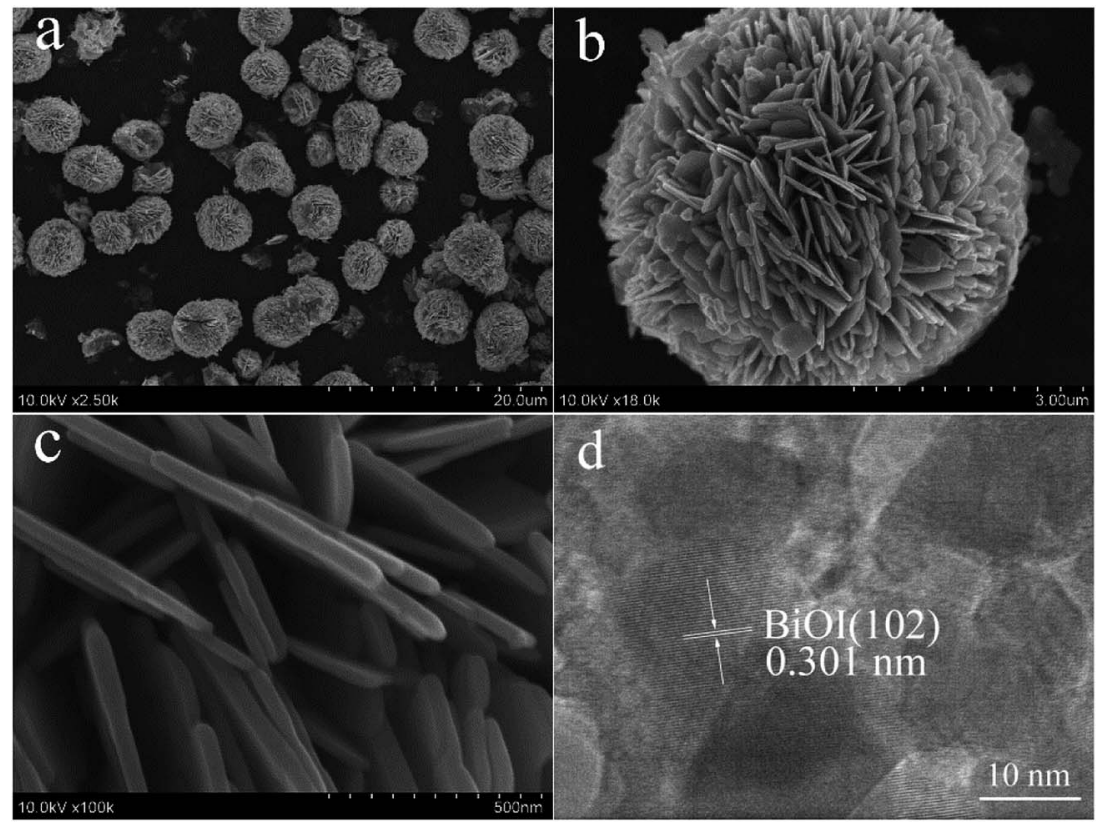

Fig. 3 (a-d) SEM images and HRTEM image of $4 \% \mathrm{P}-\mathrm{BiOl}$. 


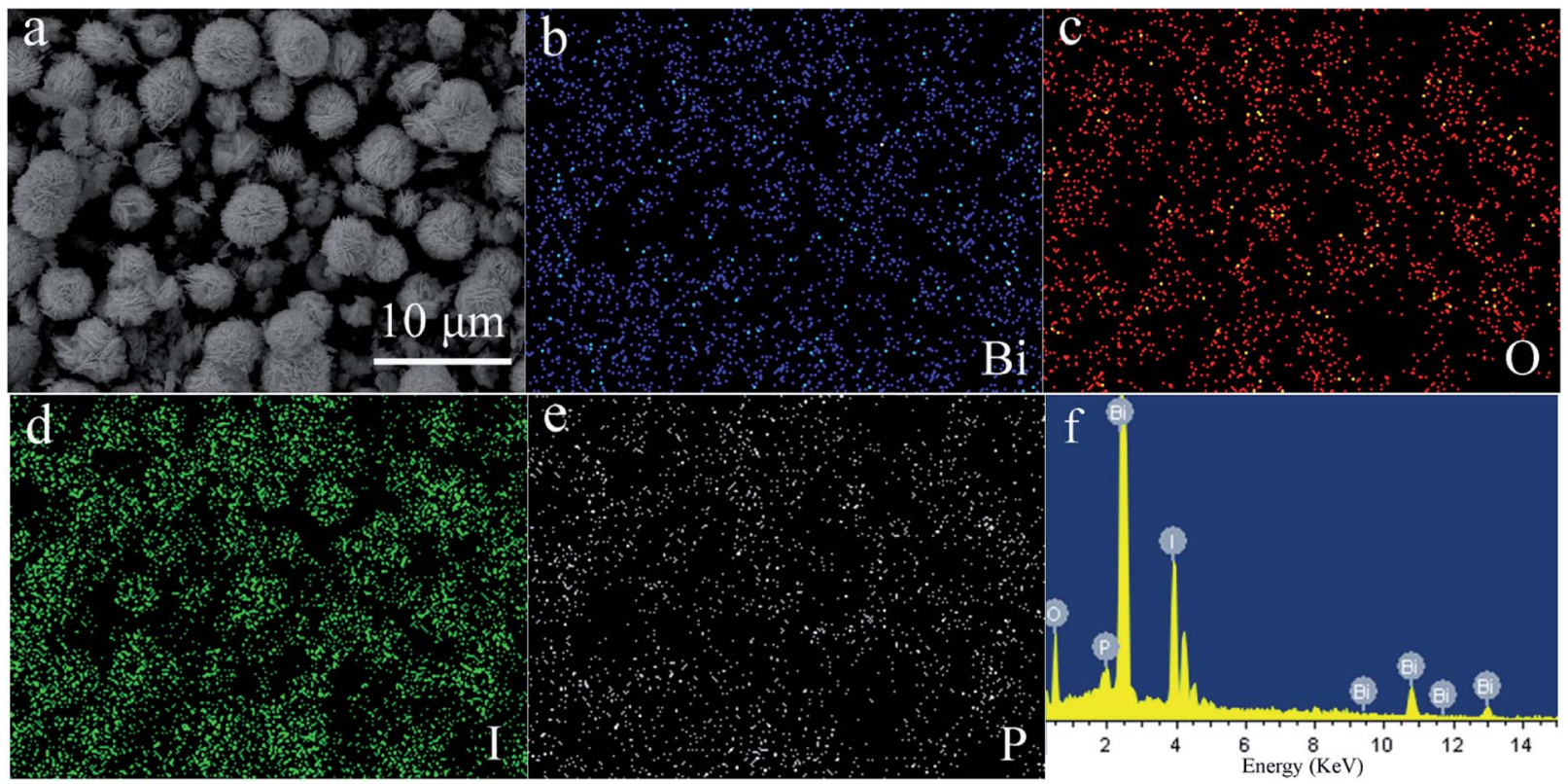

Fig. 4 SEM image (a) and EDS mapping of 4\% P-BiOl: Bi (b), O (c), I (d), P (e); EDS pattern (f) of 4\% P-BiOI.
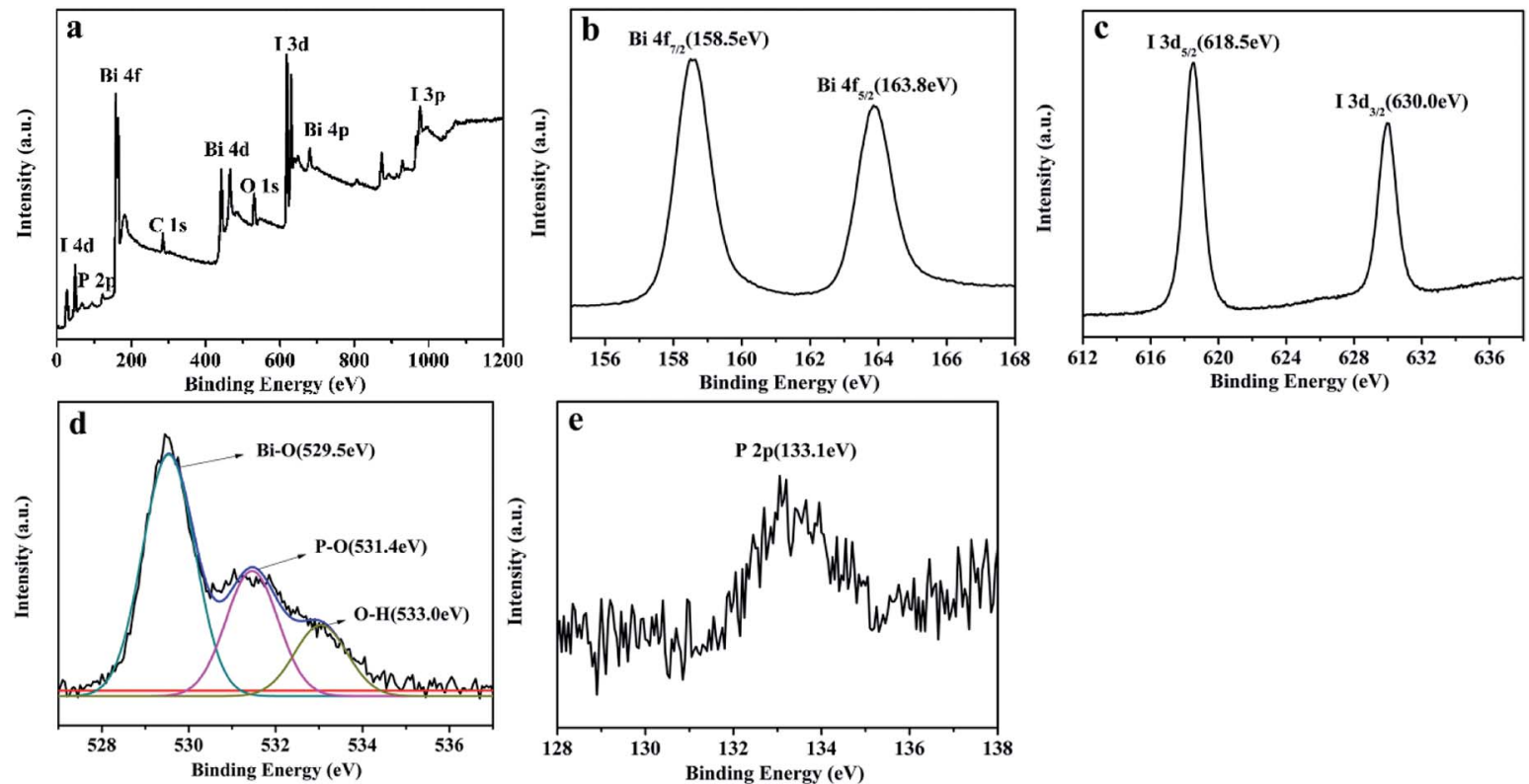

Fig. 5 XPS spectra of 4\% P-BiOl: (a) survey scan, (b) Bi 4f, (c) I 3d, (d) O 1s, (e) P 2p.

the distortion of lattice of P-doped BiOI (as the analyses of XRD patterns in Fig. 2b), we can presume that the $\mathrm{P}$ species are introduced into the lattice of BiOI, which maybe influence the photocatalytic activity of BiOI.

\subsection{FT-IR analysis}

The FT-IR spectra of as-prepared samples are shown in Fig. 6a. In the FTIR spectrum of BiOI and P-BiOI, there is a strong absorption peak at around $477 \mathrm{~cm}^{-1}$, and the absorption peak can be attributed to the stretching vibration of $\mathrm{Bi}-\mathrm{O}$ bond. ${ }^{13,30}$
In addition, there are two feeble absorption peaks at around 3564 and $1609 \mathrm{~cm}^{-1}$, which can be ascribed to the stretching and bending vibrations of $-\mathrm{OH}$ group, respectively. Compared with the FTIR spectra of BiOI and P-doped BiOI samples that is show in Fig. 6a, it can be found that there are two new absorption peaks appearing at around $1000 \mathrm{~cm}^{-1}$ and $596 \mathrm{~cm}^{-1}$ in the FTIR spectrum of P-BiOI. The absorption peak at around $1000 \mathrm{~cm}^{-1}$ and $596 \mathrm{~cm}^{-1}$ may be aroused by the stretching vibration, ${ }^{28,31,32}$ and the bending vibrations of $\mathrm{PO}_{4}{ }^{3-}{ }^{33-35}$ respectively. It could be deduced that $\mathrm{P}$ species in the $\mathrm{P}$-BiOI may be existed in the form of the pentavalent oxidation state 


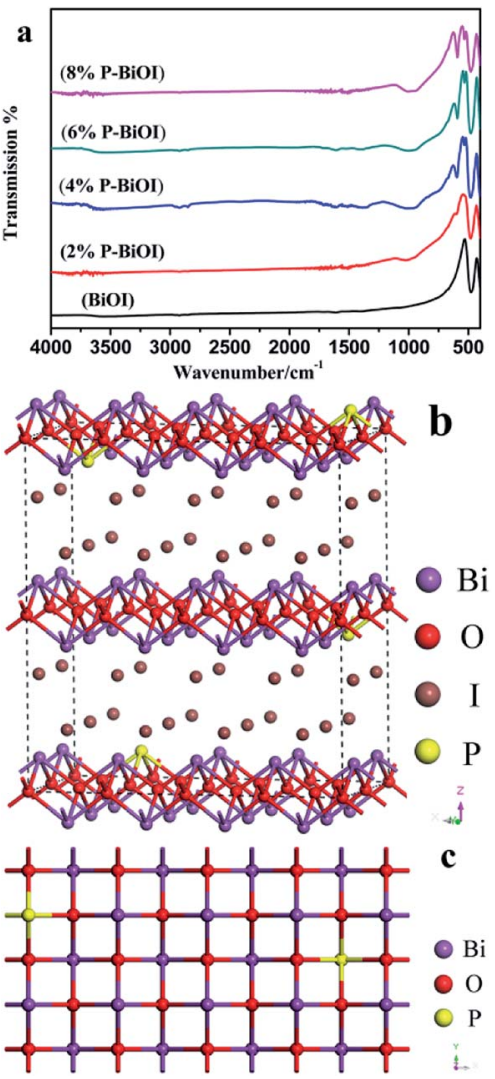

Fig. 6 (a) FTIR spectra of the samples; (b and c) the possible crystal structure of $\mathrm{P}$-doped BiOl.

$\left(\mathrm{P}^{5+}\right)$. However, it needs to be noticed that the stretching vibration of $\mathrm{P}=\mathrm{O}$ cannot be observed which appears in the range of $1300-1400 \mathrm{~cm}^{-1}{ }^{36}$ The result indicates that the $\mathrm{P}$ might exist not simply in the form of $\mathrm{PO}_{4}{ }^{3-}$, but probably in the
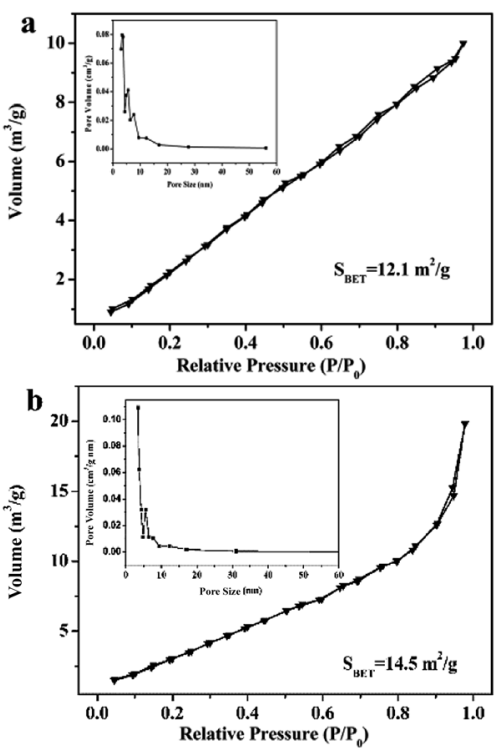

Fig. $7 \quad \mathrm{~N}_{2}$ adsorption-desorption isotherm of (a) $\mathrm{BiOl}$ and (b) $4 \% \mathrm{P}-$ BiOl. form of Bi-O-P bond. In addition, it should be emphasized that the structure and mechanism of forming bonding of $\mathrm{P}$-BiOI are still blurry. Besides, the possible crystal structure of P-BiOI is presented in Fig. $6 \mathrm{~b}$ and $\mathrm{c}$.

\section{5. $\mathbf{N}_{2}$ adsorption isotherms and pore-size distribution}

$\mathrm{N}_{2}$ adsorption-desorption isotherms and pore-size distribution are measured to determine the $S_{\mathrm{BET}}$ of the BiOI and $4 \%$ P-BiOI sample, and the results are shown in Fig. 7. All isotherms are of type IV isotherms with H3-type hysteresis loops, ${ }^{37-39}$ indicating that all the BiOI samples are mesoporous materials. The BET surface area of the $4 \% \mathrm{P}$-BiOI microspheres are measured to be $14.5 \mathrm{~m}^{2} \mathrm{~g}^{-1}$, which is slightly larger than the pure BiOI $\left(12.1 \mathrm{~m}^{2}\right.$ $\mathrm{g}^{-1}$ ). The corresponding pore-size distribution of the BiOI and P-BiOI are shown in the inset of Fig. 7, and the pore sizes of the samples are $3.4 \mathrm{~nm}$ and $3.3 \mathrm{~nm}$, respectively. The results confirm that $\mathrm{P}$ doping has less influence on the specific surfaces and the pore sizes of the BiOI sample.

\subsection{Photocatalytic activity}

The photocatalytic activities of the samples are determined by degrading the RhB dye. As shown in Fig. 8a, the photocatalytic activity of the P-BiOI sample is better than that of the pure BiOI, and $4 \%$ P-BiOI can completely degrade the dye within $40 \mathrm{~min}$ (Fig. 8b). To quantitatively understand the reaction rates of $\mathrm{RhB}$ degradation, the photocatalytic kinetic study is investigated by the pseudo-first-order model with the following equation:

$$
\ln \left(C_{0} / C\right)=k t
$$

where $C_{0}$ and $C$ are the concentrations of $\mathrm{RhB}$ at time 0 (the time to obtain adsorption-desorption equilibrium) and $t$, respectively, and $k$ is the pseudo-first-order rate constant. ${ }^{40-42}$ As seen from Fig. $8 \mathrm{c}$, the rate constants $k$ of blank, BiOI, $2 \%$ P-BiOI, $4 \%$ P-BiOI, $6 \%$ P-BiOI and $8 \%$ P-BiOI are calculated to be $1.066 \times$ $10^{-5}, 0.03234,0.05718,0.08493,0.07458$ and $0.06841 \mathrm{~min}^{-1}$, respectively. It was found that the photocatalytic activity of $4 \%$ P-BiOI sample for degradation of RhB is almost 2.6 times as high as BiOI (Fig. 8d). The photocatalytic degradation results indicate that the P doping can effectively enhance the photocatalytic performance of BiOI. The used catalyst (4\% P-BiOI) after photo-degradation of RhB was also characterized by XRD and XPS. As shown in Fig. S3, $\uparrow$ there is no significant difference observed in the XRD and XPS spectrum of $4 \%$ P-BiOI before and after the photoreaction. These results demonstrated that the structure of $4 \%$ P-BiOI has not changed before and after the photocatalytic reaction.

To evaluate reusability and stability of the pure and P-doped BiOI samples, the cycling experiments for degradation of RhB by $4 \% \mathrm{P}-\mathrm{BiOI}$ and transient photocurrent measurement of the BiOI and P-doped BiOI were carried out (Fig. 9). After four consecutive runs, the photocatalytic efficiency of $4 \%$ P-BiOI declined by $4.5 \%$ (Fig. 9a), which indicated a high stability photocatalytic activity for degradation of RhB. As shown in Fig. 9b, all photocatalysts exhibit stable photocurrent response under visible light illumination. It should be noted that the 

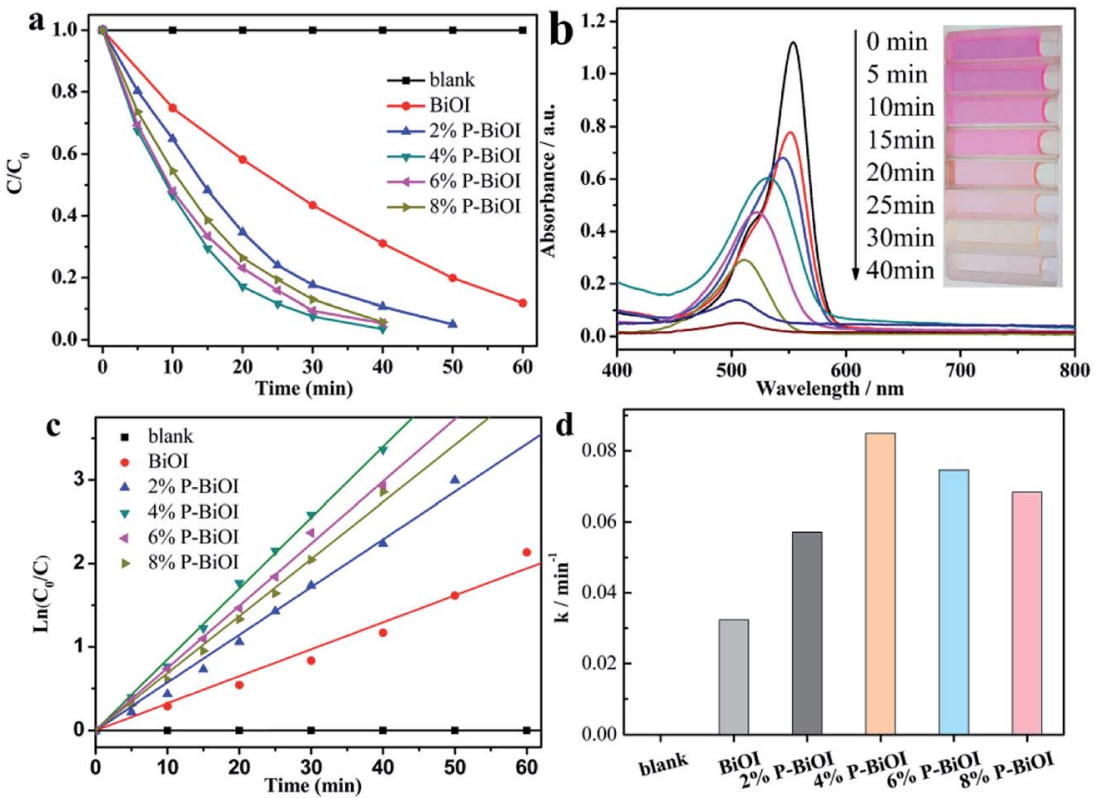

Fig. 8 (a) Photocatalytic degradation of RhB, (b) UV-vis spectral changes of RhB over $4 \% \mathrm{P}$-BiOl, (c) pseudo-first-order kinetics fitting plots and (d) apparent first-order rate constants for various photocatalysts.
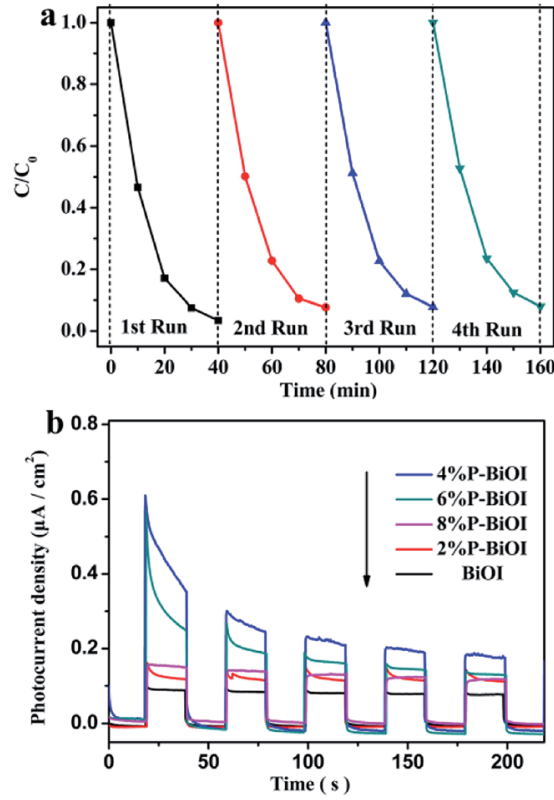

Fig. 9 (a) The cycling experiments for degradation of RhB by $4 \% \mathrm{P}-$ $\mathrm{BiOl}$; (b) transient photocurrent response of $\mathrm{BiOl}$ and $\mathrm{P}$-doped samples.

highest current is obtained from $4 \%$ P-BiOI. Thus, the obviously enhanced photocurrent response of $4 \% \mathrm{P}$-BiOI revealed the positive effect of $\mathrm{P}$ doping on facilitating the generation and separation efficiency of photoexcited charge carriers of BiOI.

\subsection{PL spectra analysis}

To investigate the separation capacity of the generated electrons and holes in the BiOI semiconductors, the PL spectra for the

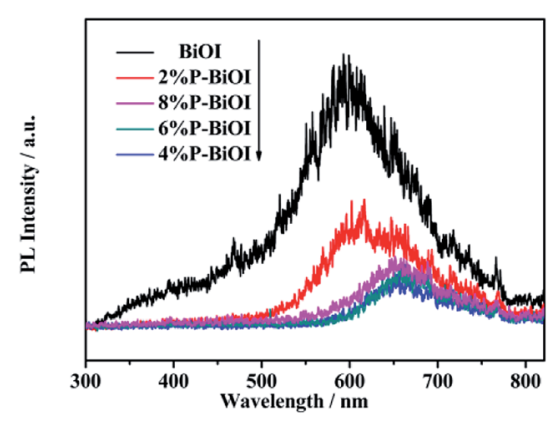

Fig. $10 \mathrm{PL}$ emission spectra of $\mathrm{BiOl}$ and $\mathrm{P}$-doped BiOl samples.

pure BiOI and P-doped BiOI samples are measured (Fig. 10). The BiOI and P-doped BiOI samples show a similar peak shape. However, the intensity of the peak in the $4 \%$ P-BiOI sample is lower than the peak in the other samples, ${ }^{43,44}$ indicating that the $4 \%$ P-BiOI sample has a more efficient separation capacity for the photogenerated carriers, which is consist with the results of the photocatalytic activities.

\subsection{Optical properties}

The UV-vis diffuse reflectance spectra of the BiOI and P-BiOI samples are shown in Fig. 11a. It can be seen that the absorption edge of the P-BiOI is similar to the pure BiOI in the visible light region. The band gap of the as-prepared samples can be measured with the following formula:

$$
\alpha h \nu=A\left(h \nu-E_{\mathrm{g}}\right)^{2}
$$

where $\alpha$ represents absorption coefficient, $h$ represents Planck constant, $\nu$ represents light frequency, $A$ represents a constant, 

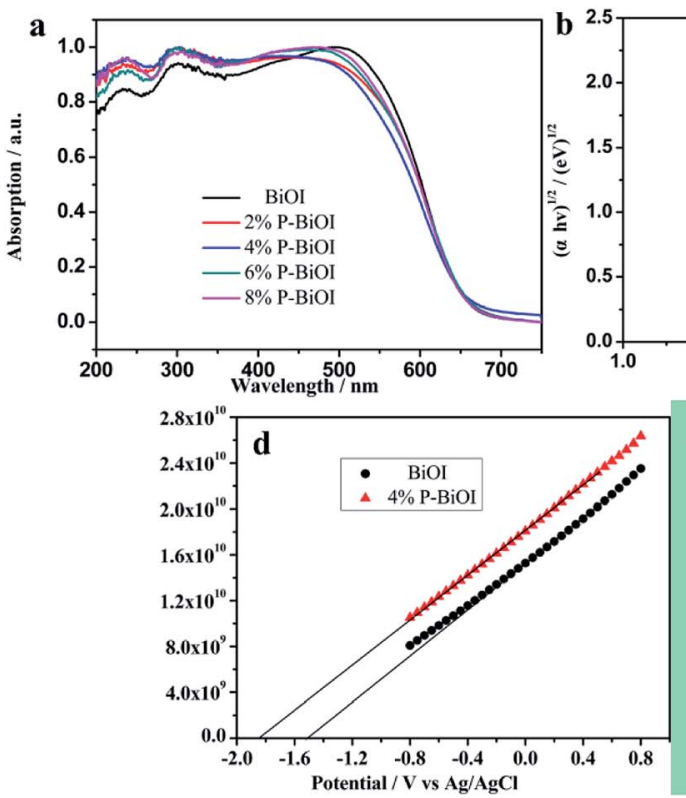
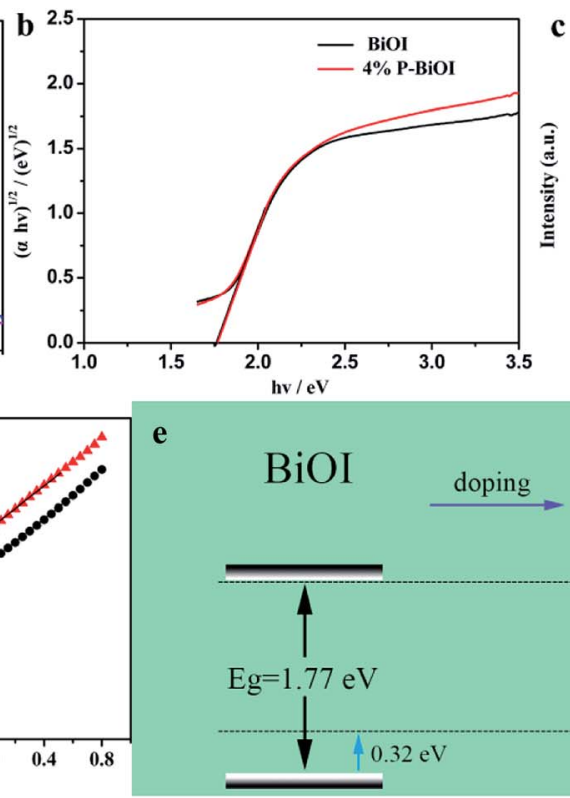

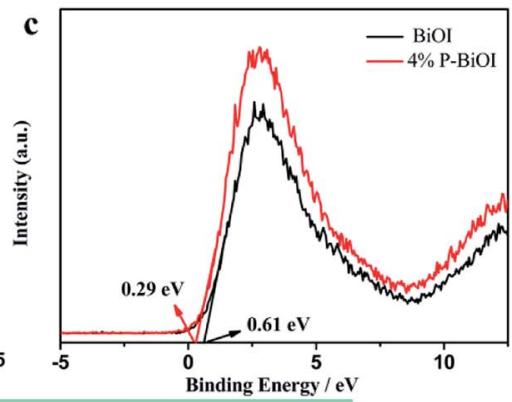

P-doped BiOI

Fig. 11 (a) UV-vis diffuse reflectance spectra, (b) the band gap energies, (c) valence-band XPS spectra and (d) Mott-Schottky plots of the samples; (e) phosphorus doping effects on the schematic of band structure evolution of BiOl.

and $E_{\mathrm{g}}$ represents band gap. ${ }^{45}$ By extrapolating to the $X$ axis (Fig. 11b), the band gap energies of BiOI and P-BiOI both were calculated to be $1.77 \mathrm{eV}$, which implies that phosphorus doping does not affect the value of the band gap.

From the standpoint of the kinetics and thermodynamic requirements of direct semiconductor photocatalytic reactions, the two features are worth noting: valence band (VB) width and conduction band (CB) minimum energy. ${ }^{46-48}$ On the one hand, the elevated VB width is advantageous for the separation of the charge carriers since the wider VB can lead to the higher mobility of the holes, which results in better photooxidation of the holes. On the other hand, the upper shift of CB should play two essential roles in photocatalytic performance: the increase in $\mathrm{CB}$ minimum not only makes the photogenerated electrons more reactive with molecular oxygen to produce superoxide ions, but also promotes photoelectron emission to the reactants results in inhibition of electron-hole recombination. The point of view is consistent with the conclusion of PL spectrum. In order to research the influence of P-doping on the VB width of BiOI, the total densities of states of XPS valence band spectra of
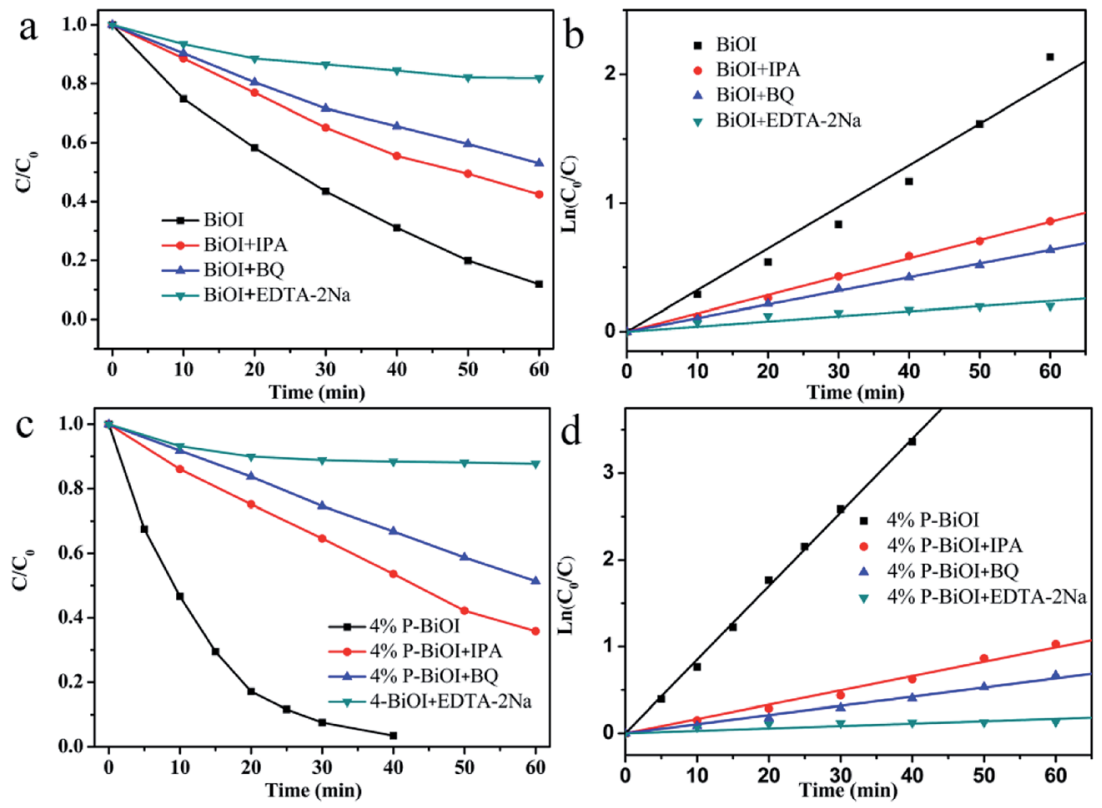

Fig. 12 (a and $c$ ) The effect of reactive groups on the photocatalytic degradation of RhB; (b and d) pseudo-first-order kinetics fitting plots. 
BiOI and P-BiOI is measured by XPS valence spectra, as shown in Fig. 11c. The pure BiOI display a valence band with the edge of the maximum energy at about $0.61 \mathrm{eV}$. In addition, in the light of the optical absorption spectrum, the value of $\mathrm{CB}$ minimum should be $-1.16 \mathrm{eV}$. Simultaneously, for the P-BiOI, the valence band maximum energy upper shifts by 0.32 to $0.29 \mathrm{eV}$ in comparison with that of pure BiOI. Combined with the consequences of optical measurements, the $\mathrm{CB}$ minimum of P-BiOI upper shifts by $0.32 \mathrm{eV}$ to $-1.48 \mathrm{eV}$ in comparison with that of pure BiOI.

To further confirm the effect of phosphorus doping on the band structure evolution of BiOI, the Mott-Schottky measurements were set to a fixed frequency of $5 \mathrm{kHz}$ and the range of $-0.8 \mathrm{eV}$ and $0.8 \mathrm{eV}$. As shown in Fig. 11d both of the samples are n-type semiconductors. ${ }^{49}$ The flat band potential $\left(E_{\mathrm{fb}}\right)$ was determined by the extrapolation of the Mott-Schottky plot, and the $E_{\mathrm{fb}}$ of BiOI and $4 \%$ P-BiOI are found to be -1.49 and $-1.83 \mathrm{~V}$ vs. $\mathrm{Ag} / \mathrm{AgCl}$ electrode $(-1.27$ and $-1.61 \mathrm{eV}$ vs. the normal hydrogen electrode, NHE), ${ }^{50,51}$ respectively. The $E_{\mathrm{fb}}$ is approximately equal to the $E_{\mathrm{CB}}$ for n-type semiconductors, which means that the conduction band position of BiOI is $0.34 \mathrm{eV}$ lower than that of $4 \%$ P-BiOI. The results of band structure calculated by Mott-Schottky fit well with XPS valence band spectra. Hence, phosphorus doping effects on the band structure evolution of BiOI as shown in Fig. 11e. Thereby, it can be speculated that the improvement of valence band width and conduction band minimum may be favourable to the enhancement of photocatalytic activity.

\subsection{Possible photocatalytic mechanism}

The degradation of organic dyes may involve one or more of the active groups such as ${ }^{\circ} \mathrm{OH}, \mathrm{h}^{+}$and ${ }^{\circ} \mathrm{O}_{2}{ }^{-} \cdot{ }^{18,45,52}$ To investigate the function of these active groups, different scavengers are added to the photocatalytic reaction system, respectively. The ethylenediaminetetraacetic acid disodium (EDTA-2Na) is used as the scavenger for $\mathrm{h}^{+}$, and the benzoquinone (BQ) is used as the scavenger for ${ }^{\circ} \mathrm{O}_{2}{ }^{-}$and the iso-propyl alcohol (IPA) is used as the scavenger for ${ }^{\circ} \mathrm{OH}$ (Fig. 12). In the case of the presence of IPA, the photocatalytic efficiency of BiOI and P-BiOI are relatively slightly reduced, indicating that the ${ }^{\circ} \mathrm{OH}$ is not significant in the photocatalytic process. When the EDTA-2Na is added to the reaction system, the photocatalytic activity of BiOI and P-BiOI are decreased significantly, suggesting that the $h^{+}$plays an indispensable role. In order to further study the effect of $\mathrm{P}$

Table 2 Pseudo-first-order rate constants for RhB photocatalytic degradation with different photocatalysts active groups

\begin{tabular}{llllll}
\hline & BiOI & & & $4 \%$ P-BiOI & \\
\cline { 2 - 3 } & $k\left(\mathrm{~min}^{-1}\right)$ & $R^{2}$ & & $k\left(\min ^{-1}\right)$ & $R^{2}$ \\
\hline Normal & $0.03234\left(k_{0}\right)$ & 0.990 & & $0.08493\left(k^{\prime}{ }_{0}\right)$ & 0.999 \\
+IPA & $0.01423\left(k_{1}\right)$ & 0.999 & & $0.01648\left(k^{\prime}{ }_{1}\right)$ & 0.995 \\
+BQ & $0.01058\left(k_{2}\right)$ & 0.999 & & $0.01054\left(k^{\prime}{ }_{2}\right)$ & 0.995 \\
+EDTA-2Na & $0.00396\left(k_{3}\right)$ & 0.962 & & $0.00280\left(k^{\prime}{ }_{3}\right)$ & 0.993
\end{tabular}

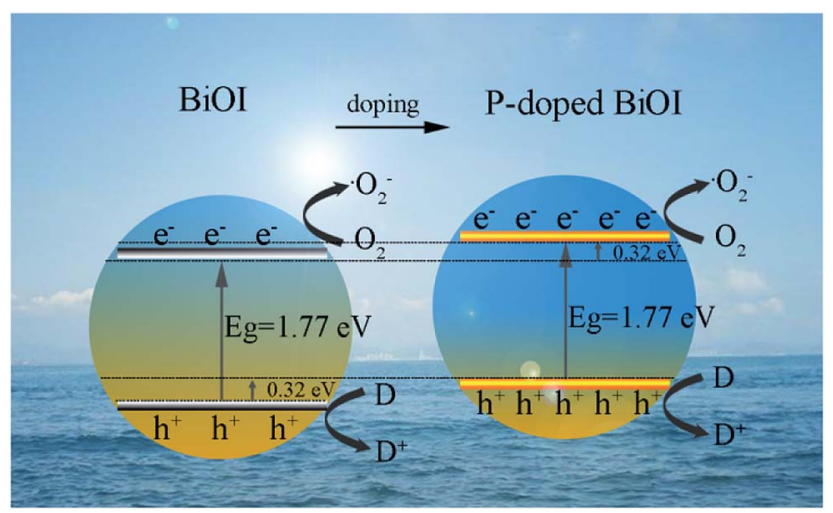

Fig. 13 Schematic illustration of the band structure of $\mathrm{BiOI}$ and $\mathrm{P}$ doped $\mathrm{BiOl}$ microspheres: the elevated $\mathrm{VB}$ maximum and rising $\mathrm{CB}$ minimum of $\mathrm{P}-\mathrm{BiOl}$ effectively separates the photoinduced electronhole pairs.

doping on photocatalytic mechanism, the rate constants $k$ of two samples by adding different scavengers are compared (Table 2). The $k_{3}^{\prime}$ is smaller than the $k_{3}$, which means that $\mathrm{h}^{+}$ plays a more important role in the photocatalytic reaction of PBiOI than that of BiOI, the result is in agreement with the literatures reported. ${ }^{53}$ Combined with XPS valence spectra data, it is concluded that the $\mathrm{P}$ doping can result in the higher mobility of holes generated which leads to the better photooxidation of holes. In addition, the $k_{2}^{\prime}$ is slightly smaller than the $k_{2}$, which implies that the $\mathrm{P}$ doping also increases the concentration of ${ }^{\circ} \mathrm{O}_{2}{ }^{-}$. As a result, the P-BiOI shows more superior photocatalytic activity than the BiOI. The effects of $\mathrm{P}$ doping on the photocatalytic mechanism of BiOI is shown in Fig. 13.

\section{Conclusions}

In summary, we had successfully synthesized 3D hierarchical microspheres via a facile hydrolytic method at room temperature. The P-BiOI microspheres were uniform in size with several micrometers and constructed by numerous 2D-nanosheets with thickness of $30-40 \mathrm{~nm}$. It was found that $\mathrm{P}$ species were introduced into the lattice of $\mathrm{BiOI}$ and formed the $\mathrm{Bi}-\mathrm{O}-\mathrm{P}$ bond. The $P$ doping not only affected the morphology and structure of BiOI, but also changed the band structure of BiOI. Most of all we found that the VB maximum and CB minimum of P-BiOI upshifts by $0.32 \mathrm{eV}$ leaded to more efficient separation capacity of the photogenerated carrier, the higher mobility of the resulting holes and the greater concentration of superoxide ions to improve the photocatalytic activity than BiOI. The change of band structure of P-BiOI was the main factor to improve the visible light photocatalytic activity.

\section{Acknowledgements}

This work was supported by the National Natural Science Foundation of China (11179029) and the Science Technology Plan Project of Hebei Province (15211109D). 


\section{References}

1 M. Kapilashrami, Y. Zhang, Y.-S. Liu, A. Hagfeldt and J. Guo, Chem. Rev., 2014, 114, 9662-9707.

2 L. Kong, C. Wang, H. Zheng, X. Zhang and Y. Liu, J. Phys. Chem. C, 2015, 119, 16623-16632.

3 N. Becknell, Y. Kang, C. Chen, J. Resasco, N. Kornienko, J. Guo, N. M. Markovic, G. A. Somorjai, V. R. Stamenkovic and P. Yang, J. Am. Chem. Soc., 2015, 137, 15817-15824.

4 K. S. Yang, Y. Dai and B. B. Huang, J. Phys. Chem. C, 2007, 111, 18985-18994.

5 C. Kang, L. Jing, T. Guo, H. Cui, J. Zhou and H. Fu, J. Phys. Chem. C, 2009, 113, 1006-1013.

6 W.-Q. Fan, X.-Q. Yu, S.-Y. Song, H.-Y. Bai, C. Zhang, D. Yan, C.-B. Liu, Q. Wang and W.-D. Shi, CrystEngComm, 2014, 16, 820-825.

7 H. Cheng, B. Huang and Y. Dai, Nanoscale, 2014, 6, 20092026.

8 X. Zhang, L. Zhang, T. Xie and D. Wang, J. Phys. Chem. C, 2009, 113, 7371-7378.

9 J. Jiang, X. Zhang, P. Sun and L. Zhang, J. Phys. Chem. C, 2011, 115, 20555-20564.

10 Y. Zhang, Q. Pei, J. Liang, T. Feng, X. Zhou, H. Mao, W. Zhang, Y. Hisaeda and X.-M. Song, Langmuir, 2015, 31, 10279-10284.

11 A. C. Mera, D. Contreras, N. Escalona and H. D. Mansilla, J. Photochem. Photobiol., A, 2016, 318, 71-76.

12 R. Hao, X. Xiao, X. X. Zuo, J. M. Nan and W. D. Zhang, J. Hazard. Mater., 2012, 209, 137-145.

13 H. L. Lin, C. C. Zhou, J. Cao and S. F. Chen, Chin. Sci. Bull., 2014, 59, 3420-3426.

14 X. Wang, S. Yang, H. Li, W. Zhao, C. Sun and H. He, RSC Adv., 2014, 4, 42530-42537.

15 X. Y. Qin, H. F. Cheng, W. J. Wang, B. B. Huang, X. Y. Zhang and Y. Dai, Mater. Lett., 2013, 100, 285-288.

16 J.-C. Wang, H.-C. Yao, Z.-Y. Fan, L. Zhang, J.-S. Wang, S.-Q. Zang and Z.-J. Li, ACS Appl. Mater. Interfaces, 2016, 8, 3765-3775.

17 C. Liao, Z. Ma, G. Dong and J. Qiu, Appl. Surf. Sci., 2014, 314, 481-489.

18 J. Liu, H. Li, N. Du, S. Song and W. Hou, RSC Adv., 2014, 4, 31393-31399.

19 J. Di, J. Xia, M. Ji, B. Wang, S. Yin, H. Xu, Z. Chen and H. Li, Langmuir, 2016, 32, 2075-2084.

20 X. Zhang and L. Z. Zhang, J. Phys. Chem. C, 2010, 114, 1819818206.

21 J. L. Liang, J. Deng, M. Li, T. Y. Xu and M. P. Tong, Colloids Surf., B, 2016, 147, 307-314.

22 M. Wang, J. Gao, G. Zhu, N. Li, R. Zhu, X. Wei, P. Liu and Q. Guo, RSC Adv., 2016, 6, 106615-106624.

23 J. L. Zhao, X. W. Lv, X. X. Wang, J. Yang, X. J. Yang and X. B. Lu, Mater. Lett., 2015, 158, 40-44.

24 H. Moradi, A. Eshaghi, S. R. Hosseini and K. Ghani, Ultrason. Sonochem., 2016, 32, 314-319.

25 Y. Zhao, X. Tan, T. Yu and S. C. Wang, Mater. Lett., 2016, 164, 243-247.
26 D. Wu, H. Wang, C. Li, J. Xia, X. Song and W. Huang, Surf. Coat. Technol., 2014, 258, 672-676.

27 J. Chen, H. B. Yang, J. Miao, H. Y. Wang and B. Liu, J. Am. Chem. Soc., 2014, 136, 15310-15318.

28 Y. Xia, Y. Jiang, F. Li, M. Xia, B. Xue and Y. Li, Appl. Surf. Sci., 2014, 289, 306-315.

29 Q. Shi, D. Yang, Z. Jiang and J. Li, J. Mol. Catal. B: Enzym., 2006, 43, 44-48.

30 Y. N. Wang, K. J. Deng and L. Z. Zhang, J. Phys. Chem. C, 2011, 115, 14300-14308.

31 F. F. Li, Y. S. Jiang, M. S. Xia, M. M. Sun, B. Xue, D. R. Liu and X. G. Zhang, J. Phys. Chem. C, 2009, 113, 18134-18141.

32 Q. Y. Wang, H. Q. Jiang, S. Y. Zang, J. S. Li and Q. F. Wang, J. Alloys Compd., 2014, 586, 411-419.

33 P. Arunkumar, C. Jayajothi, D. Jeyakumar and N. Lakshminarasimhan, RSC Adv., 2012, 2, 1477-1485.

34 V. D. Nithya, L. Vasylechko and R. K. Selvan, $R S C A d v ., 2014$, 4, 65184-65194.

35 M. H. Fulekar, A. Singh, D. P. Dutta, M. Roy, A. Ballal and A. K. Tyagi, RSC Adv., 2014, 4, 10097-10107.

36 S. Wang and S. Zhou, J. Hazard. Mater., 2011, 185, 77-85.

37 Y. Li, J. Liu, X. Huang and G. Li, Cryst. Growth Des., 2007, 7, 1350-1355.

38 A. Kay, I. Cesar and M. Grätzel, J. Am. Chem. Soc., 2006, 128, 15714-15721.

39 J. Han, G. Zhu, M. Hojamberdiev, J. Peng, X. Zhang, Y. Liu, B. Ge and P. Liu, New J. Chem., 2015, 39, 1874-1882.

40 D. Yuan, L. Huang, Y. Li, Y. Xu, H. Xu, S. Huang, J. Yan, M. He and H. Li, RSC Adv., 2016, 6, 41204-41213.

41 Y. Zhang, S. Liu, Z. Xiu, Q. Lu, H. Sun and G. Liu, J. Nanopart. Res., 2014, 16, 2375.

42 K. H. Reddy, S. Martha and K. M. Parida, Inorg. Chem., 2013, 52, 6390-6401.

43 S.-Y. Chou, C.-C. Chen, Y.-M. Dai, J.-H. Lin and W. W. Lee, RSC Adv., 2016, 6, 33478-33491.

44 H. Huang, K. Liu, Y. Zhang, K. Chen, Y. Zhang and N. Tian, RSC Adv., 2014, 4, 49386-49394.

45 Z. Yang, F. Cheng, X. Dong and F. Cui, RSC Adv., 2015, 5, 68151-68158.

46 M. Guan, C. Xiao, J. Zhang, S. Fan, R. An, Q. Cheng, J. Xie, M. Zhou, B. Ye and Y. Xie, J. Am. Chem. Soc., 2013, 135, 10411-10417.

47 G. Liu, P. Niu, C. Sun, S. C. Smith, Z. Chen, G. Q. Lu and H.-M. Cheng, J. Am. Chem. Soc., 2010, 132, 11642-11648.

48 J. Di, J. Xia, M. Ji, B. Wang, S. Yin, Q. Zhang, Z. Chen and H. Li, ACS Appl. Mater. Interfaces, 2015, 7, 20111-20123.

49 D. Kandi, S. Martha, A. Thirumurugan and K. M. Parida, J. Phys. Chem. C, 2017, 121, 4834-4849.

50 K.-W. Cheng and C.-J. Liang, Sol. Energy Mater. Sol. Cells, 2010, 94, 1137-1145.

51 M. Barawi, I. J. Ferrer, J. R. Ares and C. Sanchez, ACS Appl. Mater. Interfaces, 2014, 6, 20544-20549.

52 S. Liu, H. Liu, G. Jin and H. Yuan, $R S C A d v .$, 2015, 5, 4564645653.

53 Y. Feng, C. Liu, J. Chen, H. Che, L. Xiao, W. Gu and W. Shi, RSC Adv., 2016, 6, 38290-38299. 